Prof. Dr: Gerd Wotjak

\title{
ACERCA DEL SIGNIFICADO LÉXICO VERBAL VISTO DESDE LA LEXICOLOGÍA FUNCIONAL. INTENTO DE SÍNTESIS EN HOMENAJE A UN GRAN Y ENTRAÑABLE AMIGO
}

UNIVERSITÄT LEIPZIG 

0. La breve síntesis que nos proponemos realizar a continuación presupone el conocimiento detallado de una amplia bibliografía referente, en particular, a análisis semánticos (en la línea de la lexemática Coseriana) de verbos en una perspectiva paradigmática y sintagmática (con énfasis en la valencia lógico-semántica siguiendo a Bondzio 1971, 1993) así como de la gramática funcional de Dik y de los trabajos del homenajeado y de sus discípulos, trabajos dirigidos a la constitución de una lexicología funcional. Es obvio que no caben aquí argumentaciones pormenorizadas y que tendremos que contentarnos, en no pocos casos, con primeras hipótesis por validar aún en análisis empíricos.

0.1. Ya en los años sesenta se ha venido afirmando, en muy escasas ocasiones (cf. Koschmieder, G.F.Meier - cf. G.Wotjak 1971/1977/1979), una tendencia hacia una semantización de la sintaxis, suposición muy audaz en aquel entonces dado el aplastante predominio de la afirmación contraria promulgada por la teoría generativa transformacional y el relativo olvido del léxico como objeto de estudio lingüístico (contrastado por parte por los estudios de semántica estructural y de los campos léxico-semánticos). Esta situación ha cambiado radicalmente a partir de mediados de los años setenta convirtiéndose el léxico paulatinamente en el campo favorito o por lo menos tan legítimo como la sintaxis (cf. Steinitz 1984, Mairal 1993).

0.2. Si bien es deseable que la descripción de los fenómenos léxicos se lleve a cabo de conformidad con una determinada teoría gramatical para así dar cabida directa e inmediata a las unidades léxicas (UL) descritas dentro de la combinatoria sintagmática morfosintáctica preestablecida de la oración y el 
texto, esto no debería impedir que se tomen en consideración también aquellos análisis léxico-semánticos efectuados desde enfoques diferentes. Puede pensarse en un enfoque modular integrativo que no solo es posible sino que se aconseja para evitar trabajo doble y poder enriquecer los conocimientos acerca del enigmático y controvertido significado léxico.

0.3. Nos sentimos apoyados en nuestro afán de integrar los resultados obtenidos por el análisis de las valencias sintácticas, semánticas (lógicas) y pragmáticas, la gramática de los casos (en la línea de Fillmore), el análisis componencial de base lexemática, la semántica de las escenas o frames (cognitiva) y la lógica de predicados así como en nuestra convicción de que habría que invertir la orientación predominante en la descripción de los fenómenos sintácticos y léxicos: tomando como base la descripción semántica, es decir, partir del significado léxico y equiparar esta descripción con una descripción morfosintáctica, que, en nuestro caso, sería la de una gramática funcional (en la línea de Dik) o una gramática unificatoria (HPSG) donde se acentúa el verbo como una categoría funcional combinatoria de importancia peculiar (cf. la gramática de dependencias).

0.4. No profundizaremos en los aspectos morfosintácticos ni quisiéramos admitir, sin ulterior verificación, la plausible hipótesis de una relación isomórfica o icónica entre aspectos semántico-léxicos y aspectos morfosintáctico-combinatorios de la UL al respecto (cf. Mairal; pero también Báez San José/Penadés Martínez 1990; en contra del isomorfismo cf. Helbig 1982 y en otras muchas publicaciones).

Con esto no negamos que las divergencias y congruencias semánticas se encuentren manifestadas morfosintácticamente (en este contexto hay que reestudiar la afirmación de la lexique-grammaire de M.Gross según la cual prácticamente no hay coincidencia total entre los verbos en cuanto a su potencial distribucional-combinatorio); pero de ahí a presuponer que cualquier aspecto semántico se ve reflejado en su correspondiente combinatoria morfosintáctica o, en sentido más amplio, en el plano de la expresión (cf. el plano significante según Trujillo 1988), nos parece todavía prematuro. Nos pronunciamos, a continuación, a favor de un estudio lo más independiente posible de los fenómenos semánticos no directamente observables (que no deben fundamentarse, por miedo a caer en un círculo vicioso, recurriendo a aspectos morfosintácticos directamente observables).

1. En nuestro propio enfoque, desarrollado a partir de una semántica de corte lexemático, pasando por una complementación de esta descripción 
semántica de verbos por un análisis hasta de los elementos identificadores, integradores archisemémicos al lado de los estrictamente diferenciadores y distintivo-opositivos (semas según Pottier 1964 y Coseriu 1964 ss.), y teniendo en cuenta análisis de valencia lógico-semántica de Bondzio 1971, hemos optado por una descripción basada en la lógica de predicados (cf. Wotjak 1975, 1984; 1986, 1987, 1990, 1994). Siempre nos hemos interesado por describir la interacción inherente a las UL llamadas heteroincidentes (cf. Pottier 1971), de preferencia verbos, pero también sustantivos, adjetivos, adverbios..., entre significado léxico sistémico y paradigmático $(=$ la microestructura semántica de un verbo como parte integrante de un campo semántico-léxico determinado, es decir, una macroestructura semántica paradigmática concreta) y su potencial combinatorio sintagmático, en particular sememotáctico y en segundo lugar morfosintáctico (valencia sintáctica, secuencias actanciales, distribución morfosintáctica). En este sentido, si bien partimos de lo sistémico y paradigmático, siempre nos hemos preocupado por determinar en qué cotextos lingüísticos y contextos situacionales pueden aparecer cuántos actantes de qué características semántico-referenciales y semántico-funcionales y con qué adjuntos o circunstantes puede/suele combinarse (obligatoriamente o no) el verbo. En otras palabras, favorecemos, al igual que Mairal 1993, la constitución de un diccionario que contenga un máximo de informaciones comunicativas y que, por ello, podría considerarse un diccionario de texto o comunicativo como se ha intentado elaborar gramáticas comunicativas o discursivas. No nos parece factible, ni siquiera deseable, ubicar a nivel de diccionario la totalidad de posibles marcas o funciones comunicativas e ilocutivas/discursivas que puedan atribuírseles a las UL en el uso textual discursivo concreto; nos limitaremos, pues, a aquellas indicaciones o características/marcas semánticas, morfosintácticas y comunicativo-pragmáticas (cf. las macras diatópicas, diastráticas y diafásicas) que puedan generalizarse para el uso situacional o textual (estilística funcional de géneros de texto), reservando los usos pragmático-discursivos específicos para los análisis correspondientes del discurso y la sintaxis procedural. De esta forma, la UL con su potencial comunicativo en sus planos del contenido y de la expresión (cf. Wotjak 1994a), abarca una parte considerable de aspectos pragmático-comunicativos (mayor todavía en determinados grupos de verbos específicos como verba dicendi, etc.- cf. Mairal 1993) y morfosintácticos sistematizables. La UL, descrita desde una perspectiva funcional amplia, no puede, sin embargo, abarcar la totalidad de la combinatoria morfosintáctica ni de los usos contextuales discursivos admisibles. Funciona, más allá de un conocimiento léxico funcional y categorial, un conocimiento procedural combinatorio 
sintáctico-discursivo aparte que agruparía reglas y preferencias combinatorias válidas no solo para una determinada UL o un grupo de UL semánticamente predeterminado. Valdría la pena profundizar en lo que quedaría para tales análisis sintácticos (textuales/discursivos) si de ellos se exceptúan los aspectos morfosintácticos y pragmático-comunicativos inte grantes del potencial comunicativo de la UL respectiva.

2. Para nosotros, el significado léxico sistémico (semémico) y con ciertas modificaciones, mayormente concreciones, el significado contextual textual alosemémico de UL heteroincidentes puede equipararse a una predicación condensada, «congelada» o comprimida (cf. Greimas 1966) que puede extenderse, al pasar de esta «predicación» nuclear y virtual a una predicación real y completa, es decir, al utilizar el verbo con uno de sus significados virtuales en lo que se ha llamado una configuración oracional mínima, que solo contiene lexicalizados sus actantes, o bien en una oración expandida (con circunstantes exigidos o admitidos por el semema verbal, más eventualmente otras UL suplementarias), a formar un juicio o una aserción lógica e instanciar un proceso, un estado de cosas y establecer la referencia a hechos extralingüísticos de mundos/universos de discurso reales o ficticios.

2.1. Independientemente de Dik o de de la concepción de una four layered descripción propuesta por Mairal 1993, V.Báez San José (cf. nuevamente en 1996) y nosotros (ampliando el three level approach de Danes 1971) nos hemos pronunciado a favor de 3 ó 4 niveles de abstracción en la descripción de la interacción entre léxico y texto/discurso (cf. también la teoría de los rangos de Heger 1976; una gramática estratificacional, etc. - cf. Wotjak 1986, Wotjak 1995). Obsérvense las sorprendentes coincidencias con los niveles propuestos por Mairal (y Dik):

\subsubsection{Nivel sistémico}

a) del significado léxico virtual/potencial (=microestructura semémica) como parte del plano del contenido que abarca, además

b) la medioestructura semántica (=el conjunto de sememas adscritos a un mismo cuerpo sígnico $==>$ polisemia/homonimia) y la macroestructura semántica paradigmática (cf. campo léxico-semántico $\mathrm{y}$ otras estructuras intersemémicas) por la parte léxica lingüística y estructuras/configuraciones del conocimiento enciclopédico como las escenas, los escenarios, scripts, frames, etc. como fenómenos de la sustancia no amorfa del contenido); 
c) además, indicación de todas las características comunicativo-pragmáticas y sintácticas del plano de la expresión del signo linguiístico al respecto (= nivel de predicación nuclear).

2.1.2. Nivel sintagmático-combinatorio textual/oracional con sus macroestructuras comunicativas que comprende dos subniveles:

(i) las llamadas macroestructuras semánticas sintagmáticas (oracionales, sub-textuales) donde al llenar las «casillas vacías» preestablecidas por el significado sistémico paradigmático verbal que contiene in nuce toda la combinatoria sememotáctica y predetermina la actantificación, la combinatoria sintáctica de este verbo con co-partícipes, pasando de una predicación reducida, condensada a una predicación real y concreta, garantiza la instanciación de un determinado estado de cosas/una situación referencial.

En este complejo proceso donde suelen modificarse los sememas dando lugar a amalgamas alosemémicas específicas y donde suele poder recurrir el hablante también a inferencias sobre el conocimiento enciclopédico compartido o especializado y subjetivo, sobre las escenas y otras estructuraciones de la experiencia así como sobre la percepción de la situación referencial instanciada, conformándose así, paso a paso, un mensaje cada vez más complejo (=nivel proposicional de Mairal);

(ii) con las macroestructuras semánticas (alosemémicas) sintagmáticas no hemos descrito todavía la totalidad de los fenómenos allí observables en este nivel sintagmático y comunicativo. Tenemos que añadir los aspectos léxico-comunicativos sistémicos del nivel I así como otros aspectos comunicativos más (cf. el modo oracional y modalidad en general; marcas de certeza, probabilidad, es decir indicaciones genéricas de apreciación y actitud frente a lo dicho). Podemos hablar de macroestructuras comunicativas que abarcan, además de las macroestructuras alosemémicas indicaciones comunicativas genéricas y solo coinciden parcialmente (prácticamente todos los aspectos conservados de los niveles anteriores se ven integrados en el nivel III) con las llamadas macroestructuras ilocutivas discursivas. En el nivel II nos movemos todavía en un nivel abstracto genérico sintagmático del «texto de lengua» y no hemos llegado todavía al nivel del «texto del habla» (cf. Jäger 1986), es decir del discurso que es el del enunciado habitual y diario.

2.1.3. Nivel III = discursivo donde entran en juego el hablante con su valoración subjetiva de los hechos por designar, su intención comunicativa ilocutiva, la deixis EGO-HIC-NUNC característica del hablar. 
3. Para nosotros, el significado léxico verbal es una entidad cognitivo-conceptual sui generis que comparte elementos con la configuración o representación conceptual o del conocimiento que suele (poder) evocar/coactivar en el sistema y/o el discurso. Tal evocación del conocimiento enciclopédico puede efectuarse ya a nivel del sistema y de forma relativamente abstracta; se logra entre semema (ente lingüístico puente) y escena/script (Geschehenstyp de Klix 87) = ente cognitivo (cf. nuestras consideraciones detalladas en Wotjak 1986; 1987a y 1988), pero puede darse también en el nivel II donde junto a las diversas lexicalizaciones/actantificaciones de un verbo/ concepto como veredeln se evocan experiencias y conocimientos (tecnológicos) distintos (cf. Bäume, Stahl, Textil veredeln - cf. Wotjak 1988) compartidos (nivel II) e individuales (nivel III).

El significado léxico con su potencial designativo referencial inherente no es, pues, idéntico al concepto con que suele correlacionarse, fundamentalmente por dos razones: $1^{\circ}$, se trata de elementos cognitivos socializados y usualizados/=sememizados del conocimiento compartido por virtualmente la totalidad de los hablantes de la comunidad linguística al respecto, mientras que las representaciones del conocimiento, las macroestructuras cognitivo-conceptuales, suelen ser individuales y subjetivas o, en el caso de conceptos científicos, se trata $2^{\circ}$, de elementos referenciales cognitivos determinados en cantidad y cualidad por normalización y definición (cf. la terminología especializada). Es más, el significado léxico suele abarcar, más allá de los elementos cognitivos compartidos y en no pocos casos perspectivados, focalizados, pues sememizados de forma algo divergente entre las lenguas y culturas (cf. Wotjak 1987), también otros elementos semémicos, ya no más identificadores o integradores, sino diferenciadores o modificadores que distinguen los significados en una perspectiva intralingual e interlinguísticas. Tales elementos, entre ellos los semas connotativos, de evaluación, emoción, etc. socializados y usualizados ellos también, forman parte del significado léxico e incluso le transfieren su carácter idiosincrático, pero no suelen darse en absoluto o, tal vez, solo en forma individual-subjetiva, en las conceptualizaciones cognitivas, en las macroestructuras cognitivas consociadas en el enunciado.

3.1. En nuestra opinión, el significado léxico referencial-denotativo que evoca una conceptualización individual, una parte del conocimiento enciclopédico genérico o especializado adquirido por el hablante en cuestión y sujeta a permanentes cambios, asegura cierta estabilidad comunicativa y funciona como una especie de denominador común y punto de referencia sincrónicamente bastante invariable disponible para la virtual totalidad de hablantes 
de esta misma comunidad linguística. Se asemeja a una llave más o menos genérica, una «llave maestra» que permite abrir las puertas detrás de las cuales se encuentra abritado y almacenado nuestro conocimiento enciclopédico personal. El significado léxico es, pues, premisa para la comunicación siendo, al mismo tiempo, resultado almacenado, memorizado de actos comunicativos anteriores. En este contexto, el significado puede compararse a un patrón, un módulo de conducta o de acción (cf. Wotjak 1987a; Jena); es como un Gebrauchsbedingungstyp (cf. Brekle 1963 y las condiciones de uso de Leisi 1961) y, en cierta forma, podría decirse que, en el caso de la semántica, se trata de una pragmática socializada y usualizada (cf. Wotjak 1987 b y de forma casi idéntica Langacker 1987).

3.2. En el significado léxico verbal distinguimos entre el núcleo denotativo referencial identificador o integrador común para varias UL integrantes de un mismo campo y llamado «proposición básica archisemémica» y los modificadores. Tal núcleo funge como una dirección electrónica, un denominador archisemémico común que garantiza que el semema en cuestión puede compararse a otros sememas conteniendo este mismo denominador, esta misma proposición básica (PB). Nos parece que el archisemema como conceptualización común para un determinado campo léxico-semántico, una macroestructura semántica paradigmática dada, se repite más o menos por completo e invariable en estas proposiciones básicas nucleares del significado léxico de distintas UL integrantes del campo.

Las PB, análogamente a la descripción de los semas diferenciadores y consideradas como componentes recurrentes, pueden descomponerse en elementos recurrentes - pasando por proposiciones básicas parciales a proposiciones o predicados nucleares elementales con un alto grado de recurrencia (cf. (1a-1c).

(1a) Fórmula archisemémica especial FAEI del DESPLAZAMIENTO/ representación de la proposición básica PB1-FAE1

[ADESSE (x\&z, LOC1)]ti-TP1=SETTING \& [OPER(x,z)\&CAUS(z,(ADESSE(x\&z,LOC2))]ti+k-TP2=EVENT \& [ADESSE/x\&z, LOC3)]ti+l-TP3=CONSEQ

Especificación semántico-funcional de los argumentos de los functores/ predicados elementales:

$\mathrm{x}=\mathrm{TP} 1=\mathrm{LOCG} ; \mathrm{TP} 2=$ BENUTZER \& LOCG; TP3= LOCG

$\mathrm{z}=\mathrm{TP} 1=\mathrm{LOCG} ; \mathrm{TP} 2=$ INSTRUMENT \& CAUSATOR; TP3 $=$ LOCG

ejemplos en alemán: fahren, reiten... 
(1a') Er fährt mit dem Auto in die Stadt.

(1a") Er fährt mit dem Auto seines Freundes in die Stadt.

(1a")Er fährt das Auto in die Garage.

(En todos los ejemplos el Er conduce él mismo)

(1b) [ADESSE(x,z) \& ADESSE(x\&z, LOC1)]ti-TP1-SETTING \& [OPER $(x, z) \&$ CAUS $(x \& z,(A D E S S E(x \& z, L O C 2))] \mathrm{ti}+k-T P 2-E V E N T \&$ [ADESSE $(x \& z$, LOC3)]ti+l-TP3-CONSEQ

$\mathrm{x}=\mathrm{TP} 1=\mathrm{LOCG} ; \mathrm{TP} 2=\mathrm{BENUTZER} \& \mathrm{CO}-\mathrm{CAUSATOR} /$ Réalisateur;TP3 $=\mathrm{LOCG}$ $\mathrm{z}=\mathrm{TP} 1=\mathrm{LOCP} \& \mathrm{LOCG} ; \mathrm{TP} 2=$ INSTRUMEN \&CO-CAUSATOR;TP3=LOCG ejemplos del alemán con fahren:

(1b') Er fährt mit dem Fahrrad in die Stadt.

(1b") Er fährt mit dem Fahrrad seines Freundes in die Stadt.

(1b"')?Er fährt das Fahrrad in die Garage. (eher bringt)

(En todos los tres ejemplos Er aparece como CO-CAUSATOR y

Réalisateur donde se impone una interpretación de AGENte para $\mathrm{x}$ ).

(1c) [OPER(x,z)\&ADESSE(x\&y,z)\&ADESSE(x\&y\&z,LOC1)]ti-TP1 \& [CAUS(z, (ADESSE(x\&y\&z, LOC2))]ti+k-TP2 \&

[ADESSE $(x \& y \& z$, LOC3]ti+l-TP3=CONSEQ

$\mathrm{x}=\mathrm{TP} 1=\mathrm{BENUTZER} \& \mathrm{LOCG} ; \mathrm{TP} 2=\mathrm{LOCG} ; \mathrm{TP} 3=\mathrm{LOCG}$

$\mathrm{y}=\mathrm{TP} 1=\mathrm{LOCG} ; \mathrm{TP} 2=\mathrm{LOCG} ; \mathrm{TP} 3=\mathrm{LOCG} \longrightarrow$ PATIENS/BEFÖRDERTER

$\mathrm{z}=\mathrm{TP} 1=\mathrm{INSTRUMEN} \&$ LOC \&LOCG;TP2=CAUSATOR\&LOCG;TP3=LOCG ejemplos con fahren 2:

(1c') Er fährt mit dem Bus in die Stadt.

(1c") Er fährt mit dem Schiff nach Dänemark.

(1c") Er fährt mit dem Auto seines Freundes (mit) in die Stadt.

3.3. Ya que en este aspecto nuestro enfoque se diferencia bastante del concebido por Leocadio Martín Mingorance y practicado por sus discípulos, especificaremos estos aspectos de estructuración interna del significado algo más. Podemos destacar que los predicados semánticos constitutivos (cf. ADESSE, OPER) requieren una determinada cantidad (generalmente 2) y calidad de argumentos, que, desde la perspectiva de la PB global, reciben, como potencial argumental, sus características semántico-denotativo-clasemáticas y funcionales (=los casos llamados semánticos, los theta-roles de Chomsky 1981). Así, el significado léxico sistémico determinado por análisis paradigmáticos conlleva ya predeterminaciones de orden sintagmático 
bastante detalladas. Postulamos que estos argumentos de la proposición básica constituyen las casillas vacías por llenar en la combinatoria sememotáctica y, a fin de cuentas, también la combinatoria morfosintáctica, la secuencia actancial. Cuando las UL como co-partícipes de la situación, del evento, proceso, acción, etc., designados por el semema verbal que evoca a menudo una escena o un Geschehenstyp cognitivo almacenados, entran en la combinatoria morfosintáctica del verbo llenando las casillas vacías semémicamente predeterminadas, hablamos de actantes o de actantificaciones que se efectuan en base a los argumentos subyacentes. Solo cuando se lexicalizan los argumentos - conformándose en esto a las características categoriales clasemáticas y funcionales adscritas al potencial argumental semémico - podemos hablar de actantes; de forma que se determina la calidad de ser actante para una UL en la combinatoria morfosintáctica del verbo por referencia a un argumento dado en la PB del semema verbal correspondiente. Si no se corresponden, esto significa que no se trata de actantes, sino de un circunstante o bien de una UL más allá de la combinatoria sememotáctica $y$, por ende, también valencial morfosintáctica. No puede exceder el número de actantes el de los argumentos; pero no en todas las macroestructuras semánticas sintagmáticas oracionales deben aparecer todas las actantificaciones virtualmente admisibles. Hay más; puede haber restriciones combinatorias actantificadoras para determinados argumentos - debidas a su carácter peculiar (cf. los argumentos «constantes» en B. Wotjak 1990) o a condiciones discursivo-comunicativas que aconsejan la no actantificación.

3.3.1. Para ilustrar con algunos ejemplos cómo interactúan el semema /léxico y la enunciación, veamos los ejemplos siguientes:

(2) *La besó con sus labios.-La besó con sus labios sangrientos. La besó. Sus labios eran sangrientos. — Su beso la hizo sobresaltarse.

(3) No lo sé. No sé; no sé si vendrá mañana.

(4) Espero estación 8.15 (p.m.) Saludos Enrique (telegrama)

Mientras que en (2) funciona un mecanismo de bloqueo interno semémico, en (3) la omisión de actantes se explica por razones comunicativas (o de preferencia del género de texto y necesidad de economía = $=>$ telegrama). Si el lo eliminado se presupone conocido, no debe aparecer (cf. elipsis comunicativas situacionales). Lo mismo es válido para (4) donde la econo- 
mía nos impulsa a reducir la comunicación a lo estrictamente indispensable: el destinatario del telegrama, que es una contestación a otro anterior recibido (o a una carta), sabe que lo/la espera Enrique en la estación de trenes a las 8.15 horas por la tarde/noche.

En (2) nos enfrentamos, sin embargo, a hechos inherentes al semema mismo: podemos postular que forma parte constitutiva del la PB de besar, además de los argumentos variables de AGENTE y DESTINATARIO, también un argumento constante INSTRUMENTO especificado aquí por labios; argumento que solo en posición transfrástica podrá actantificarse sin adjetivo que le especifique como es de rigor al querer actantificarlo intrafrásticamente.

3.3.2. La teoría de la valencia sintáctica siempre ha hablado de actantes obligatorios (este concepto de «obligatoriedad» ha tenido que revisarse y especificarse - cf. Pasch 1977, Helbig 1982, 1992, Storrer 1996) y facultativos de un lado y los adjuntos (libres) o circunstantes del otro. Se ha utilizado una multitud de métodos operativos para poder definir cuándo se trata de actantes y cuándo de circunstantes (cf. Helbig 1982, 1992) y justamente a la vista de estos procedimientos el propio Helbig ha reforzado su tesis de la no isomorfía entre lo sintáctico y lo semántico. Nos parece que en comparación con estos métodos aparentemente formales o con el criterio de la necesidad comunicativa sugerido por Andresen 1983, el criterio meramente semántico ofrecido por nuestro enfoque garantiza mayor objetividad.

3.3.3. Podemos deducir aún mayor información de tal descripción detallada del significado léxico: no solo puede ilustrarse la cantidad (y calidad funcional, la atribución de casos semánticos a partir de los predicados semánticos genéricos y recurrentes y desde la perspectiva de la PB global) de los argumentos y posibles correspondencias semémicas para otros co-partícipes del verbo en las configuraciones oracionales extendidas, sino que también podrá correlacionarse con mayor certeza a las macroestructuras cognitivas coactivadas (cf. el Geschehenstyp mencionado en 5). No podemos detallar aquí, sin embargo, los problemas con que nos tropezamos al fijar cuáles de los co-partícipes de una escena, un script o Geschehenstyp pasan a ser argumentos y cuáles pasan a ser modificadores y cuáles no pasan del todo a ser sememizados (cf. Wotjak 1994; 1986 y el ejemplo de un Geschehenstyp bajo 5).

(5) Presentación de una situación cognitiva-designada/Geschehenstyp (apud Klix 1987) = situación referencial instanciable/instanciada 
clínica

LOC médico (cirujano)

HT1 (portador de una acción)

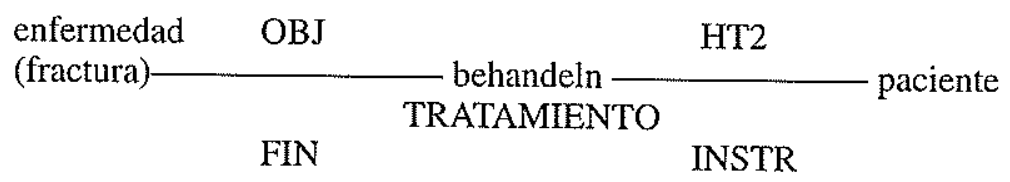

diagnóstico intervención bisturí

(narkosis)

3.3.4. Si no excluimos por completo cualquier referencia de lo estrictamente semémico a lo sintáctico-combinatorio, en un moderado isomorfismo o presunta iconicidad, resulta más fácil determinar cuáles son los actantes (el resto, por default procedure, serían circunstantes).

3.4. El significado léxico, además de los elementos constitutivos de la $\mathrm{PB}$ (predicados semánticos y argumentos), abarca también los elementos diferenciadores sememizados, es decir, socializados y usualizados, ellos también y llamados modificadores (cf. ya Bondzio 1971). Desde el enfoque semántico, nos parece posible correlacionar los circunstantes en la combinatoria sintáctica con estos modificadores al igual que se han correlacionado los actantes con los argumentos subyacentes.

3.5. Podría llegarse, quizá, también a resultados convincentes, sin tales análisis componenciales pormenorizados, como lo demuestran los análisis efectuados por los discípulos de Leocadio en sus descripciones de los predicate frames. A título de ejemplo para lo que pasa al afinar y detallar el análisis, véanse las posibles simbolizaciones del concepto del DESPLAZAMIENTO:

(a) MOVE ( $x$ ) O DELOC ( $x$ )

(b) X STARE/ADESSE LOC1 $\rightarrow$ X ADESSE LOC2

(c) cf. las indicaciones detalladas bajo (l)

Hemos diferenciado entre fórmulas archisemémicas genéricas y fórmulas archisemémicas específicas (para más detalles cf. también B.Wotjak/ G.Wotjak 1995). 
3.6. De lo expuesto se desprende que, si bien diferenciamos entre significado y designación en el sentido que les da Coseriu (cf. también Trujillo 1988), los dos conceptos se ven como estrechamente interactuantes: podemos designar gracias al potencial referencial y designativo contenido en las PB del núcleo semémico de verbos, es decir, el significado léxico contiene elementos de designación que posibilitan la designación la cual, sin embargo, no es idéntica al significado. No podemos ni debemos evitar el correlato extralingüístico referencial (sea este cognitivo o no), lo que Coseriu ha reprochado al análsis componencial efectuado por Pottier 1964 con las denominaciones parasilla diciendo que está demasiado impregnado por el mundo de las cosas. Ello, no solo en el caso de los sustantivos concretos autoincidentes investigados, sino también en el caso de las UL heteroincidentes, difícilmente podrá evitarse. Lo que importa en tal caso es aprovechar todos los recursos lingüísticos para poder establecer los elementos constitutivos del archisemema en el sentido demostrado de una (re)construcción de un denominador común archisemémico, de la PB identificadora y archisemémica entre los verbos de una misma dimensión, de un mismo campo o microcampo.

4. Nos parece que el enfoque aquí esbozado que es el resultado de años de interés por la semántica léxica se presta bastante bien para completar los análisis de una lexicología funcional naciente y que brinda una posibilidad de entender mejor la interacción entre léxico/semántica y gramática, pero también entre el léxico paradigmático y el eje sintagmático y la combinatoria de las UL inmanente a los significados léxicos de las UL heteroincidentes.

Para las UL autoincidentes fallan tales consideraciones, lo cual no significa que no puedan o deban descomponerse ellas también. Se podrán detectar elementos integrantes y en común aquí también, los que garantizan cierta estructuración del léxico (y del saber enciclopédico). Podrán señalarse, incluso, ciertas conceptualizaciones genéricas - parecidas a las archisemémicas - en las que se enmarcan estos sustantivos en concordancia con los estados de cosas y las experiencias designativas y comunicativas adquiridas (cf. Irsula 1994).

Como pensamos haber señalado, en lo semántico importa menos la categoría morfosintáctica que la distinción poco conocida y aprovechada, pero esencialmente semántica entre UL autoincidentes (que se refieren a sí mismas) y heteroincidentes, es decir, que se refieren como sememas relacionales a por lo menos 1 , generalmente 2 términos como polos de tal relación. Dado que puede haber sustantivos heteroincidentes (y no solo los infinitivos sustantivados o deverbativos, sino también otros como, por ej., amistad), la distinción no se restringe a las categorías sustantivo y verbo. 


\section{BIBLIOGRAFÍA}

Andresen, H. 1973: Ein methodischer Beitrag zur Unterscheidung von Ergänzung und Angabe im Rahmen der Valenztheorie. En: Deutsche Sprache $1,1-32$.

Báez San José, V./Penadés Martínez, I. 1990: Diccionario informatizado de construcciones oracionales y el proyecto «Esquemas sintátctico-semánticos del español». En: G.Wotjak/A.Vega (eds.): La Descripción del verbo español. Anexo 32 a Verba. Santiago de Compostela: Universidad, 33-70.

Báez San José, V. 1996: Acerca de una clasificación de los adverbios. En G.Wotjak (ed.): Adverbios y circunstantes en el español actual. Tübingen: Narr (en imprenta).

Bondzio, W. 1971: Valenz, Bedeutung und Satzmodelle. En: G.Helbig: Beiträge zur Valenztheorie. Halle:Niemeyer, 85-103.

Bondzio, W. 1974: Die Valenz zweiter Stufe als Grundlage der Adverbialsyntax. En: Wissenschaftliche Zeitschrift der Humboldt-Universität zu Berlin. Ges.-Sprachwiss. R. 23. Berlin, 245-259.

Bondzio, M. 1993: Funktorenstrukturen in der deutschen Sprache. Ein Beitrag zur Grundlegung einer semantischen Valenztheorie. En: F.Simmler (ed.): Probleme der funktionellen Grammatik. Berlin/Bern/Frankfurt/New York/Paris/Wien: Peter Lang, 21-88.

Brekle, H.E. 1963: Semantische Analyse con Wertadjektiven als Determinanten persönlicher Substantive in W. Caxton's Prologen und Epilogen. Tübingen:Narr.

Coseriu, E. 1964: Pour une sémantique diachronique structurale. En: Travaux de littérature et de Linguistique de Strasbourg II/1, 139-186.

Danes, F. 1971: A three- level Approach to Syntax. En: Travaux linguistiques de Prague 1. Praga, 225-240.

Dik, S.C. 1979: A Theory of Functional Grammar. Amsterdam.

Greimas, A.J. 1966: Sémantique structurale. Paris: Larousse.

Heger, K. 1976: Monem, Wort, Satz und Text. Tübingen: Niemeyer.

- 1991: Überlegungen zur Gegenüberstellung von «Actant» und «Circonstant». En: Sprachtheorie und Theorie der Sprachwissenschaft. Festschrift für R. Engler zum 60. Geburtstag. Tübingen: Narr, 118-133.

Helbig, G./Schenkel, W. 1975: Wörterbuch zur Valenz und Distribution 
deutscher Verben. Leipzig: Enzyklopädie (2a ed.).

Helbig, G. 1982: Valenz-Satzglieder-semantische Kasus-Satzmodelle. Leipzig: Enzyklopädie.

- 1992: Probleme der Valenz- und Kasustheorie. Tübingen: Niemeyer.

Irsula Peña, J. 1994: Substantiv-Verb-Kollokationen. Kontrastive Untersuchungen Deutsch-Spanisch. Frankfurt/Berlin...:Lang.

Jäger, G. 1986: Bedeutung und Text. En: Übersetzungswissenschaftliche Beiträge. No. IX. Leipzig, 1-66.

Klix, F. 1987: On the role of knowledge in sentence comprehension. En: Preprints of the Plenary Session Papers (XIVth International Congress of Linguists). Berlin, 11-124.

Kotschi, T. 1991: Zirkumstanten und komplexe Prädikate. En: P. Koch/T. Krefeld (eds.): Connexiones Romanicae. Tübingen: Niemeyer, 129-138.

Langacker, R.W. 1987: Foundations of Cognitive Grammar. Vol. 1. Standford: Univ. Press.

Leisi, E. 1961: Der Wortinhalt. Heidelberg: Quelle.

Mairal Usón, R. 1993: Complementation Patterns of Cognitive, Physical Perception and Speech Act Verbs in the English Language. A FunctionalCognitive Approach. Tesis doctoral. Universidad de Zaragoza 1993.

Martín Mingorance, L. 1984: Lexical Fields and Stepwise Lexical Decomposition in a Contrastive English-Spanish Verb Valency Dictionary. En: R.R.K.Hartmann (ed.): Lexeter '83 Proceedings. Tübingen: Niemeyer, 226-236.

- 1987: Classematics in a Functional-Lexematic Grammar of English. En: Actas del X Congreso de la Asociación Española de Estudios Anglo-Norteamericanos. Zaragoza. Universidad, 377-382.

- 1990: Functional Grammar and Lexematics in lexicography. En: J.Tomaszczyk/B.Lewandowska-Tomaszczyk (eds.): Meaning and Lexicography. Amsterdam/Philadelphia: Benjamins, 227-253.

Pasch, R. 1977: Zum Status der Valenz. En: Linguistische Studien, R. A, 42. Berlin, 1-50.

Pottier, B. 1964: Vers une sémantique moderne. En: Travaux de littérature et de Linguistique de Strasbourg II/1, 107-136.

- 1971: Gramática del español. Madrid. 
- 1974: La linguistique. Paris.

Raskin, V. 1985: Script-based Semantics: a brief outline. En: Quaderni di semantica, No. 2. Bologna, 306-313. 101-106. Once again on scripts. En:Quaderni di semantica, No. 1. Bologna,
101-106.

Steinitz, R. 1984: Lexikalisches Wissen und die Struktur von LexikonEinträgen. En: Linguistische Studien, R.A, 116. Berlin, 1-88.

Storrer, A. 1996: Wie notwendig sind obligatorische Valenzstellen? Faktoren der Weglaßbarkeit von Valenzstellen im Text. En: G. Gréciano/H. Schumacher (eds.): Lucien Tesnière - Syntaxe structurale et opérations mentales. Tübingen: Niemeyer, 225-238.

Tesnière, L. 1959: Eléments de syntaxe structurale. Paris.

Trujillo, R. 1976: Elementos de semántica lingüística. Madrid.

- 1988: Introducción a la semántica española. Madrid.

Welke, K. 1988: Einführung in die Valenz- und Kasustheorie. Leipzig.

Wotjak, B. 1982: Untersuchungen zur Inhalts-und Ausdrucksstruktur ausgewählter deutscher Verben des Beförderns. Berlin (=Linguistische Studien 103).

- 1990: Acerca de incorporaciones lexemáticas en verbos españoles. En: G. Wotjak/A. Vega (eds.): La descripción del verbo español. Anexo 32 a Verba. Universidade de Santiago de Compostela, 259-264.

—/ Wotjak, G. 1995: Werben für Verben? En: H. Popp (hg.): Deutsch als Fremdsprache. An den Quellen eines Faches. München: iudicium, 235-286.

Wotjak, G. 1971/77/79: Untersuchungen zur Struktur der Bedeutung. Berlin: Akademie-Verlag (1. y 2a. edición aumentada). Investigaciones sobre la estructura del significado. Madrid:Gredos 1979.

- 1975: Valenzmodelle und semantische Merkmalanalyse. En: Leipziger Linguistische Arbeitsberichte (LAB) 11, 19-39.

- 1984: Zur Aktantifizierung von Argumenten ausgewählter deutscher Verben. En: Zeitschrift für Germanistik 4. Leipzig, 401-414.

- 1985: Zur Vertextung von Argumenten ausgewählter deutscher Verben. En: Grammatik im Unterricht, ed. K. Nyholm. Turku, 199-210.

- 1986: Zu den Interrelationen von Bedeutung, Mitteilungsgehalt, kommunikativem Sinn und kommunikativem Wert. En: Übersetzungswis- 
senschaftliche Beiträge. No. IX. Leipzig, 67-127.

- 1987: La sémantique lexicale: état actuel et perspectives. En: Linguistische Studien, No. 166. Berlin, 45-85.

- 1987a: Bedeutung und Wissenskonfiguration - Eine Quadratur des Kreises, oder wie löst man den gordischen Knoten? En: Zeitschrift für Germanistik, No.6. Leipzig, 698-708.

- 1987b: Illokutives, Pragmatisches und Semantisches - Pragmatisches im Semantischen? En: Lunder Germanistische Forschungen 55. Lund, 127137.

- 1988: Verbbedeutung und Geschehenstypbeschreibung. En: Zeitschrift für Psychologie. No. 4. Leipzig, 325-334.

- 1990: Fundamentos metodológicos para una descripción modular integrativa del potencial comunicativo de los verbos. En: G. Wotjak/A. Vega (eds.): La descripción del verbo español. Anexo 32 a Verba. Universidade de Santiago de Compostela, 265-285.

- 1991: Einige Ergänzungen und Angaben zu Ergänzungen und Angaben. En: P. Koch/T. Krefeld (eds.): Connexiones Romanicae. Tübingen: Niemeyer, 109-128.

- 1994: Actantes y sus correspondencias semánticas en verbos. En: A. Endruschat/M. Vilela/G. Wotjak (eds.): Sintaxis y semántica de los verbos en el español y el portugués de hoy. Oporto: Editorial Universitaria (en imprenta).

- 1994a: Acerca del potencial comunicativo de las unidades léxicas. En: Voz y Letra, V/1, 155-173.

- 1995: Equivalencia semántica, equivalencia comunicativa y equivalencia translémica. En: Hieronymus, No.1. Madrid: Universidad Complutense. 93-112. 